\title{
Genus-1 Virasoro conjecture along quantum volume direction
}

\author{
Xiaobo Liu *
}

\begin{abstract}
In this paper, we show that the derivative of the genus-1 Virasoro conjecture for GromovWitten invariants along the direction of quantum volume element holds for all smooth projective varieties. This result provides new evidence for the Virasoro conjecture.
\end{abstract}

\section{Introduction}

The Virasoro conjecture predicts that the generating functions of the Gromov-Witten invariants of smooth projective varieties are annihilated by a sequence of differential operators which form a half branch of the Virasoro algebra. This conjecture was proposed by Eguchi-Hori-Xiong [EHX] and modified by S. Katz [CX]. In case the underlying manifold is a point, this conjecture is equivalent to Witten's conjecture [W], proved by Kontsevich [K], that the generating function of intersection numbers on the moduli spaces of stable curves is a $\tau$-function of the KdV hierarchy. Together with Tian, we proved that the genus-0 part of the Virasoro conjecture holds for all compact symplectic manifolds (cf. [LT]). For manifolds with semisimple quantum cohomology, the genus-1 part of this conjecture was proved by Dubrovin and Zhang [DZ]. Without assuming semisimplicity, the genus-1 Virasoro conjecture was studied in [L1] and [L2]. Among other results, it was proved in [L1] that the genus-1 Virasoro conjecture can be reduced to the the $L_{1}$-constraint. Using the genus-1 topological recursion relation, it was also proved that Virasoro constraints can be reduced to equations on the small phase space, i.e. the space of cohomology classes of the underlying manifold. Compatibility conditions for Virasoro conjectures were studied in [L2]. Despite these efforts, the general case of the genus-1 Virasoro conjecture is still largely open. In this paper, we give more evidence to the genus-1 Virasoro conjecture without any assumption on the quantum cohomology of the underlying manifold.

Let $M$ be a smooth projective variety. Choose a basis $\left\{\gamma_{\alpha} \mid \alpha=1, \ldots, N\right\}$ of the space of cohomology classes $H^{*}(M ; \mathbb{C})$. For simplicity, we assume $H^{\text {odd }}(M ; \mathbb{C})=0$. We choose the basis in such a way that $\gamma_{1}$ is the identity of the cohomology ring and $\gamma_{\alpha} \in H^{p_{\alpha}, q_{\alpha}}(M)$ for some integers $p_{\alpha}$ and $q_{\alpha}$. Let $\left\{t^{1}, \ldots, t^{N}\right\}$ be the coordinates on $H^{*}(M ; \mathbb{C})$ with respect to this basis. We can identify each $\gamma_{\alpha}$ with the vector field $\frac{\partial}{\partial t^{\alpha}}$ and further identify each cohomology class with a constant vector field on $H^{*}(M ; \mathbb{C})$. Let

$$
b_{\alpha}=p_{\alpha}-\frac{1}{2}(d-1)
$$

where $d$ is the complex dimension of $M$. Then the Euler vector field (on the small phase space) is defined to be

$$
E:=c_{1}(M)+\sum_{\alpha}\left(b_{1}+1-b_{\alpha}\right) t^{\alpha} \gamma_{\alpha}
$$

${ }^{*}$ Research was partially supported by NSF grant DMS-0905227. 
We refer to [LiT] RT] for definitions of Gromov-Witten invariants. In genus-1 case, it suffices to study only primary Gromov-Witten invariants since all genus-1 descendant invariants can be reduced to primary invariants due to the genus- 1 topological recursion relation. Therefore we only consider primary Gromov-Witten invariants in this paper. Let $F_{g}$ be the generating function of genus- $g$ primary Gromov-Witten invariants of $M$. The $k$-point function is defined to be

$$
\left\langle\left\langle v_{1} \cdots v_{k}\right\rangle_{g}:=\sum_{\alpha_{1}, \ldots, \alpha_{k}} f_{\alpha_{1}}^{1} \cdots f_{\alpha_{k}}^{k} \frac{\partial^{k} F_{g}}{\partial t^{\alpha_{1}} \cdots \partial t^{\alpha_{k}}}\right.
$$

for vector fields $v_{i}=\sum_{\alpha} f_{\alpha}^{i} \gamma_{\alpha}$ where $f_{\alpha}^{i}$ are functions on $H^{*}(M ; \mathbb{C})$. Note that $F_{g}$ and $\left\langle\langle\cdots\rangle_{g}\right.$ in this paper corresponds to $F_{g}^{s}$ and $\left\langle\langle\cdots\rangle_{g, s}\right.$ in [L1]. Let $\eta_{\alpha \beta}=\int_{M} \gamma_{\alpha} \cup \gamma_{\beta}$ be the intersection form on $H^{*}(M, \mathbb{C})$. We will use $\eta=\left(\eta_{\alpha \beta}\right)$ and $\eta^{-1}=\left(\eta^{\alpha \beta}\right)$ to lower and raise indices. For example $\gamma^{\alpha}:=\eta^{\alpha \beta} \gamma_{\beta}$ where repeated indices should be summed over entire range. We recall that the quantum product of two vector fields $v_{1}$ and $v_{2}$ is defined by

$$
v_{1} \circ v_{2}:=\left\langle\left\langle v_{1} v_{2} \gamma^{\alpha}\right\rangle_{0} \gamma_{\alpha}\right.
$$

Define

$$
\Psi:=\left\langle\left\langle E^{2}\right\rangle\right\rangle_{1}+\frac{1}{24} \sum_{\alpha}\left\langle\left\langle E E \gamma_{\alpha} \gamma^{\alpha}\right\rangle\right\rangle_{0}-\frac{1}{2} \sum_{\alpha}\left(b_{\alpha}\left(1-b_{\alpha}\right)-\frac{b_{1}+1}{6}\right)\left\langle\left\langle\gamma_{\alpha} \gamma^{\alpha}\right\rangle\right\rangle_{0}
$$

where $E^{2}=E \circ E$ is the quantum square of the Euler vector field. It was proved in [L1] that, for any smooth projective variety $M$, the genus-1 Virasoro conjecture can be reduced to a single equation on $H^{*}(M ; \mathbb{C})$ :

$$
\Psi=0 .
$$

Moreover, since $E \Psi=\Psi$ (cf. [L1, Lemma 6.3]), the genus-1 Virasoro conjecture holds if and only if

$$
E \Psi=0
$$

Therefore, to prove the genus- 1 Virasoro conjecture, it suffices to show that $v \Psi=0$ for all vector field $v$ on $H^{*}(M ; \mathbb{C})$. It follows from the string equation that $\gamma_{1} \Psi=0$ where $\gamma_{1}=E^{0}$ is the identity of the ordinary cohomology ring. In this paper we will give another vector field which always annihilates $\Psi$.

Define the vector field

$$
\Delta:=\gamma^{\alpha} \circ \gamma_{\alpha}
$$

If, in the definition of $\Delta$, we replace the quantum product "o" by the ordinary cup product, we get a vector field proportional to the volume element. Therefore we call $\Delta$ the quantum volume element. The main result of this paper is the following

Theorem 1.1 For all smooth projective varieties,

$$
\Delta \Psi=0 .
$$

This result provides a new evidence for the genus-1 Virasoro conjecture. 


\section{Properties of Euler vector fields}

We first recall some basic properties of the Euler vector field $E$. We start with the quasihomogeneity equation

$$
\langle\langle E\rangle\rangle_{g}=(3-d)(1-g) F_{g}+\frac{1}{2} \delta_{g, 0} \sum_{\alpha, \beta} \mathcal{C}_{\alpha \beta} t_{0}^{\alpha} t_{0}^{\beta}-\frac{1}{24} \delta_{g, 1} \int_{M} c_{1}(M) \cup c_{d-1}(M) .
$$

This equation is a consequence of the divisor equation. Define the grading operator $G$ by

$$
G(v):=\sum_{\alpha} b_{\alpha} f_{\alpha} \gamma_{\alpha}
$$

for any vector field $v=\sum_{\alpha} f_{\alpha} \gamma_{\alpha}$. Derivatives of quasi-homogeneity equation has the form

$$
\begin{aligned}
\left\langle\left\langle E v_{1} \cdots v_{k}\right\rangle_{g}=\right. & \sum_{i=1}^{k}\left\langle\left\langle v_{1} \cdots G\left(v_{i}\right) \cdots v_{k}\right\rangle_{g}\right. \\
& -(2 g+k-2)\left(b_{1}+1\right)\left\langle\left\langle v_{1} \cdots v_{k}\right\rangle_{g}\right. \\
& +\delta_{g, 0} \nabla_{v_{1}, \cdots, v_{k}}^{k}\left(\frac{1}{2} \mathcal{C}_{\alpha \beta} t_{0}^{\alpha} t_{0}^{\beta}\right)
\end{aligned}
$$

where $\mathcal{C}_{\alpha \beta}$ is defined by $c_{1}(M) \cup \gamma_{\alpha}=\mathcal{C}_{\alpha}^{\beta} \gamma_{\beta}$, and $\nabla$ is the trivial connection on $H^{*}(M ; \mathbb{C})$ defined by $\nabla \gamma_{\alpha}=0$ for all $\alpha$. In particular,

$$
\left\langle\left\langle E v_{1} v_{2} \gamma^{\alpha}\right\rangle_{0} \gamma_{\alpha}=G\left(v_{1}\right) \circ v_{2}+v_{1} \circ G\left(v_{2}\right)-G\left(v_{1} \circ v_{2}\right)-b_{1} v_{1} \circ v_{2} .\right.
$$

Combining with [L1, Lemma 4.2], we can obtain

$$
\begin{aligned}
\nabla_{E^{k}} \Delta= & \left\langle\left\langle E^{k} \gamma^{\alpha} \gamma_{\alpha} \gamma^{\beta}\right\rangle\right\rangle_{0} \gamma_{\beta} \\
= & \left(k-b_{1}\right) E^{k-1} \circ \Delta-G\left(E^{k-1} \circ \Delta\right)-\sum_{i=1}^{k-1} \Delta \circ E^{i-1} \circ G\left(E^{k-i}\right) \\
& -\sum_{i=1}^{k-1} G\left(\Delta \circ E^{i-1}\right) \circ E^{k-i}
\end{aligned}
$$

for $k \geq 1$. Covariant derivative of $E$ is given by

$$
\nabla_{v} E=-G(v)+\left(b_{1}+1\right) v .
$$

Using the fact that

$$
\nabla_{w}\left(v_{1} \circ v_{2}\right)=\left(\nabla_{w} v_{1}\right) \circ v_{2}+v_{1} \circ\left(\nabla_{w} v_{2}\right)+\left\langle\left\langle w v_{1} v_{2} \gamma^{\alpha}\right\rangle_{0} \gamma_{\alpha},\right.
$$

we can also show that

$$
\nabla_{\Delta} E^{2}=\Delta \circ G(E)-G(\Delta) \circ E-G(\Delta \circ E)+\left(b_{1}+2\right) \Delta \circ E .
$$

Combining equations (8) and (10), we have

$$
\left[E^{2}, \Delta\right]=-2 b_{1} E \circ \Delta-2 G(E) \circ \Delta .
$$




\section{Proof of the main theorem}

For any vector fields $v_{1}, \ldots v_{4}$ on the small phase space, we define

$$
\begin{aligned}
G_{0}\left(v_{1}, v_{2}, v_{3}, v_{4}\right)=\sum_{g \in S_{4}} \sum_{\alpha, \beta} & \frac{1}{6}\left\langle\left\langle v_{g(1)} v_{g(2)} v_{g(3)} \gamma^{\alpha}\right\rangle\right\rangle_{0}\left\langle\left\langle\gamma_{\alpha} v_{g(4)} \gamma_{\beta} \gamma^{\beta}\right\rangle\right\rangle_{0} \\
& +\frac{1}{24}\left\langle\left\langle v_{g(1)} v_{g(2)} v_{g(3)} v_{g(4)} \gamma^{\alpha}\right\rangle\right\rangle_{0}\left\langle\left\langle\gamma_{\alpha} \gamma_{\beta} \gamma^{\beta}\right\rangle\right\rangle_{0} \\
& \left.-\frac{1}{4}\left\langle\left\langle v_{g(1)} v_{g(2)} \gamma^{\alpha} \gamma^{\beta}\right\rangle\right\rangle_{0}\left\langle\left\langle\gamma_{\alpha} \gamma_{\beta} v_{g(3)} v_{g(4)}\right\rangle\right\rangle_{0}\right\},
\end{aligned}
$$

and

$$
\begin{aligned}
G_{1}\left(v_{1}, v_{2}, v_{3}, v_{4}\right)= & \sum_{g \in S_{4}} 3\left\langle\left\langle\left\{v_{g(1)} \circ v_{g(2)}\right\}\left\{v_{g(3)} \circ v_{g(4)}\right\}\right\rangle\right\rangle_{1} \\
& -\sum_{g \in S_{4}} 4\left\langle\left\langle\left\{v_{g(1)} \circ v_{g(2)} \circ v_{g(3)}\right\} v_{g(4)}\right\rangle\right\rangle_{1} \\
& -\sum_{g \in S_{4}} \sum_{\alpha}\left\langle\left\langle\left\{v_{g(1)} \circ v_{g(2)}\right\} v_{g(3)} v_{g(4)} \gamma^{\alpha}\right\rangle\right\rangle_{0}\left\langle\left\langle\gamma_{\alpha}\right\rangle\right\rangle_{1} \\
& +\sum_{g \in S_{4}} \sum_{\alpha} 2\left\langle\left\langle v_{g(1)} v_{g(2)} v_{g(3)} \gamma^{\alpha}\right\rangle\right\rangle_{0}\left\langle\left\langle\left\{\gamma_{\alpha} \circ v_{g(4)}\right\}\right\rangle\right\rangle_{1} .
\end{aligned}
$$

Note that $G_{0}$ is completely determined by genus- 0 data, while each term in $G_{1}$ contains genus- 1 information. These two tensors are connected by Getzler's equation (cf. Ge]):

$$
G_{0}+G_{1}=0
$$

Theorem 1.1 is obtained by applying this equation to $v_{1}=v_{2}=E, v_{3}=\gamma^{\alpha}, v_{4}=\gamma_{\alpha}$, and summing over $\alpha$.

We first consider the genus-1 part of Equation (11).

\section{Lemma 3.1}

$$
\sum_{\alpha} G_{1}\left(E, E, \gamma^{\alpha}, \gamma_{\alpha}\right)=24 \Delta\left\langle\left\langle E^{2}\right\rangle\right\rangle_{1}
$$

Proof: We will use the convention that repeated indices should be summed over their entire range. Therefore we will omit $\sum_{\alpha}$ in the left hand of this formula. To compute $G_{1}\left(E, E, \gamma^{\alpha}, \gamma_{\alpha}\right)$, we notice that

$$
\begin{aligned}
\left\langle\left\{E \in \gamma_{\alpha}\right\}\left\{\gamma^{\alpha} \circ E\right\}\right\rangle_{1} & =\left\langle\left\langle E \gamma_{\alpha} \gamma^{\beta}\right\rangle\right\rangle_{0}\left\langle\left\langle E \gamma^{\alpha} \gamma^{\mu}\right\rangle\right\rangle_{0}\left\langle\left\langle\gamma_{\beta} \gamma_{\mu}\right\rangle_{1}\right. \\
& =\left\langle\left\langle E E \gamma_{\alpha}\right\rangle\right\rangle_{0}\left\langle\left\langle\gamma^{\alpha} \gamma^{\beta} \gamma^{\mu}\right\rangle\right\rangle_{0}\left\langle\left\langle\gamma_{\beta} \gamma_{\mu}\right\rangle_{1}\right. \\
& =\left\langle\left\langle\left\{E^{2} \circ \gamma^{\mu}\right\} \gamma_{\mu}\right\rangle\right\rangle_{1}=\left\langle\left\langle\left\{E^{2} \circ \gamma^{\alpha}\right\} \gamma_{\alpha}\right\rangle\right\rangle_{1} .
\end{aligned}
$$

In the second equality, we have used the associativity of the quantum product. This observation 
enables us to simplify the formula for $G_{1}\left(E, E, \gamma^{\alpha}, \gamma_{\alpha}\right)$ and obtain

$$
\begin{aligned}
& G_{1}\left(E, E, \gamma^{\alpha}, \gamma_{\alpha}\right) \\
= & 24\left\langle\left\langle E^{2} \Delta\right\rangle\right\rangle_{1}-48\left\langle\langle\{E \circ \Delta\} E\rangle_{1}-4\left\langle\left\langle E^{2} \gamma^{\alpha} \gamma_{\alpha} \gamma^{\beta}\right\rangle\right\rangle_{0}\left\langle\left\langle\gamma_{\beta}\right\rangle\right\rangle_{1}\right. \\
& -16\left\langle\left\langle\left\{E \circ \gamma^{\alpha}\right\} \gamma_{\alpha} E \gamma^{\beta}\right\rangle\right\rangle_{0}\left\langle\left\langle\gamma_{\beta}\right\rangle\right\rangle_{1}-4\left\langle\left\langle\Delta E E \gamma^{\beta}\right\rangle\right\rangle_{0}\left\langle\left\langle\gamma_{\beta}\right\rangle\right\rangle_{1} \\
& +24\left\langle\left\langle E E \gamma^{\alpha} \gamma^{\beta}\right\rangle\right\rangle_{0}\left\langle\left\langle\left\{\gamma_{\alpha} \circ \gamma_{\beta}\right\}\right\rangle\right\rangle_{1}+24\left\langle\left\langle E \gamma_{\alpha} \gamma^{\alpha} \gamma^{\beta}\right\rangle\right\rangle_{0}\left\langle\left\langle\left\{\gamma_{\beta} \circ E\right\}\right\rangle_{1} .\right.
\end{aligned}
$$

We now use formulas in Section 2 to compute each term on the right hand side of this equation. Using equation (10), we have

$$
\begin{aligned}
\left\langle\left\langle E^{2} \Delta\right\rangle\right\rangle_{1} & =\Delta\left\langle\left\langle E^{2}\right\rangle\right\rangle_{1}-\left\langle\left\langle\left\{\nabla_{\Delta} E^{2}\right\}\right\rangle\right\rangle_{1} \\
& =\Delta\left\langle\left\langle E^{2}\right\rangle\right\rangle_{1}-\left\langle\left\langle\left\{\Delta \circ G(E)-G(\Delta) \circ E-G(\Delta \circ E)+\left(b_{1}+2\right) \Delta \circ E\right\}\right\rangle_{1} .\right.
\end{aligned}
$$

Since $\langle\langle E\rangle\rangle_{1}$ is a constant due to the quasi-homogeneity equation, by equation (9), we have

$$
\begin{aligned}
\langle\langle\{E \circ \Delta\} E\rangle\rangle_{1} & =\{E \circ \Delta\}\langle\langle E\rangle\rangle_{1}-\left\langle\left\langle\left\{\nabla_{E \circ \Delta} E\right\}\right\rangle\right\rangle_{1} \\
& =\left\langle\left\langle\left\{G(E \circ \Delta)-\left(b_{1}+1\right) E \circ \Delta\right\}\right\rangle_{1} .\right.
\end{aligned}
$$

By equation (8), we have

$$
\left\langle\left\langle E^{2} \gamma^{\alpha} \gamma_{\alpha} \gamma^{\beta}\right\rangle\right\rangle_{0}\left\langle\left\langle\gamma_{\beta}\right\rangle_{1}=\left\langle\left\langle\left\{\left(2-b_{1}\right) E \circ \Delta-G(E \circ \Delta)-G(E) \circ \Delta-E \circ G(\Delta)\right\}\right\rangle_{1} .\right.\right.
$$

By equation (7), we have

$$
\begin{aligned}
& \left\langle\left\langle\left\{E \circ \gamma^{\alpha}\right\} \gamma_{\alpha} E \gamma^{\beta}\right\rangle\right\rangle_{0}\left\langle\left\langle\gamma_{\beta}\right\rangle_{1}\right. \\
= & \left\langle\left\langle\left\{G\left(E \circ \gamma^{\alpha}\right) \circ \gamma_{\alpha}+E \circ \gamma^{\alpha} \circ G\left(\gamma_{\alpha}\right)-G(E \circ \Delta)-b_{1} E \circ \Delta\right\}\right\rangle_{1} .\right.
\end{aligned}
$$

As a convention, we arrange the basis $\left\{\gamma_{1}, \ldots, \gamma_{N}\right\}$ of $H^{*}(M, \mathbb{C})$ in such a way that the degree $p_{\alpha}+q_{\alpha}$ of $\gamma_{\alpha} \in H^{p_{\alpha}, q_{\alpha}}$ is non-decreasing with respect to $\alpha$ and if two cohomology classes have the same dimension, we also require that the holomorphic dimension $p_{\alpha}$ is non-decreasing. Under this convention, we have

$$
G\left(\gamma^{\alpha}\right)=\left(1-b_{\alpha}\right) \gamma^{\alpha}
$$

for all $\alpha$, and

$$
G\left(\gamma^{\alpha}\right) \circ \gamma_{\alpha}=\Delta-\gamma^{\alpha} \circ G\left(\gamma_{\alpha}\right)
$$

On the other hand,

$$
G\left(\gamma^{\alpha}\right) \circ \gamma_{\alpha}=\eta^{\alpha \beta} G\left(\gamma_{\beta}\right) \circ \gamma_{\alpha}=G\left(\gamma_{\beta}\right) \circ \gamma^{\beta}=\gamma^{\alpha} \circ G\left(\gamma_{\alpha}\right) .
$$

So we must have

$$
G\left(\gamma^{\alpha}\right) \circ \gamma_{\alpha}=\gamma^{\alpha} \circ G\left(\gamma_{\alpha}\right)=\frac{1}{2} \Delta .
$$

Hence

$$
\begin{aligned}
G\left(E \circ \gamma^{\alpha}\right) \circ \gamma_{\alpha} & =\left\langle\left\langle E \gamma^{\alpha} \gamma^{\beta}\right\rangle\right\rangle_{0} G\left(\gamma_{\beta}\right) \circ \gamma_{\alpha}=G\left(\gamma_{\beta}\right) \circ\left(E \circ \gamma^{\beta}\right) \\
& =\frac{1}{2} E \circ \Delta .
\end{aligned}
$$


Therefore we obtain

$$
\left\langle\left\langle\left\{E \circ \gamma^{\alpha}\right\} \gamma_{\alpha} E \gamma^{\beta}\right\rangle\right\rangle_{0}\left\langle\left\langle\gamma_{\beta}\right\rangle\right\rangle_{1}=\left\langle\left\langle\left\{\left(1-b_{1}\right) E \circ \Delta-G(E \circ \Delta)\right\}\right\rangle_{1} .\right.
$$

Similarly,

$$
\left\langle\left\langle\Delta E E \gamma^{\beta}\right\rangle\right\rangle_{0}\left\langle\left\langle\gamma_{\beta}\right\rangle_{1}=\left\langle\left\langle\left\{G(\Delta) \circ E+\Delta \circ G(E)-G(\Delta \circ E)-b_{1} \Delta \circ E\right\}\right\rangle_{1},\right.\right.
$$

and

$$
\begin{aligned}
& \left\langle\left\langle E E \gamma^{\alpha} \gamma^{\beta}\right\rangle\right\rangle_{0}\left\langle\left\langle\left\{\gamma_{\alpha} \circ \gamma_{\beta}\right\}\right\rangle_{1}\right. \\
= & \left\langle\left\langle\left\{\gamma_{\alpha} \circ\left(G(E) \circ \gamma^{\alpha}+E \circ G\left(\gamma^{\alpha}\right)-G\left(E \circ \gamma^{\alpha}\right)-b_{1} E \circ \gamma^{\alpha}\right)\right\}\right\rangle_{1}\right. \\
= & \left\langle\left\langle\left\{G(E) \circ \Delta-b_{1} E \circ \Delta\right\}\right\rangle_{1} .\right.
\end{aligned}
$$

To compute the last term in equation (12), we first compute

$$
\begin{aligned}
\left\langle\left\langle E \gamma_{\alpha} \gamma^{\alpha} \gamma^{\beta}\right\rangle\right\rangle_{0} \gamma_{\beta} & =G\left(\gamma_{\alpha}\right) \circ \gamma^{\alpha}+\gamma_{\alpha} \circ G\left(\gamma^{\alpha}\right)-G(\Delta)-b_{1} \Delta \\
& =\left(1-b_{1}\right) \Delta-G(\Delta)
\end{aligned}
$$

by equation (13). So the last term in equation (12) is

$$
\left\langle\left\langle E \gamma_{\alpha} \gamma^{\alpha} \gamma^{\beta}\right\rangle\right\rangle_{0}\left\langle\left\langle\left\{\gamma_{\beta} \circ E\right\}\right\rangle\right\rangle_{1}=\left\langle\left\langle\left\{\left(1-b_{1}\right) E \circ \Delta-E \circ G(\Delta)\right\}\right\rangle\right\rangle_{1} .
$$

After plugging the above formulas into equation (12), all terms on the right hand side cancel except the term $24 \Delta\left\langle\left\langle E^{2}\right\rangle\right\rangle_{1}$. The lemma is thus proved.

Now we consider the genus-0 part of Equation (11). Let

$$
\Phi:=-\frac{1}{24} \sum_{\alpha}\left\langle\left\langle E E \gamma_{\alpha} \gamma^{\alpha}\right\rangle\right\rangle_{0}+\frac{1}{2} \sum_{\alpha}\left(b_{\alpha}\left(1-b_{\alpha}\right)-\frac{b_{1}+1}{6}\right)\left\langle\left\langle\gamma_{\alpha} \gamma^{\alpha}\right\rangle\right\rangle_{0} .
$$

Then

$$
\Psi=\left\langle\left\langle E^{2}\right\rangle\right\rangle_{1}-\Phi
$$

and the genus-1 Virasoro conjecture can be reduced to

$$
\left\langle\left\langle E^{2}\right\rangle\right\rangle_{1}=\Phi
$$

\section{Lemma 3.2}

$$
\sum_{\alpha} G_{0}\left(E, E, \gamma^{\alpha}, \gamma_{\alpha}\right)=-24 \Delta \Phi
$$

Proof: Again we will assume that repeated indices will be summed over their entire range. First, by definition of $G_{0}$, we have

$$
\begin{aligned}
G_{0}\left(E, E, \gamma^{\alpha}, \gamma_{\alpha}\right)= & 2\left\langle\left\langle E E \gamma^{\alpha} \gamma^{\beta}\right\rangle\right\rangle_{0}\left\langle\left\langle\gamma_{\beta} \gamma_{\alpha} \gamma^{\mu} \gamma_{\mu}\right\rangle\right\rangle_{0}+2\left\langle\left\langle E \gamma^{\alpha} \gamma_{\alpha} \gamma^{\beta}\right\rangle\right\rangle_{0}\left\langle\left\langle\gamma_{\beta} E \gamma^{\mu} \gamma_{\mu}\right\rangle_{0}\right. \\
& +\left\langle\left\langle E E \gamma^{\alpha} \gamma_{\alpha} \Delta\right\rangle\right\rangle_{0}-2\left\langle\left\langle E E \gamma^{\beta} \gamma^{\mu}\right\rangle\right\rangle_{0}\left\langle\left\langle\gamma_{\beta} \gamma_{\mu} \gamma^{\alpha} \gamma_{\alpha}\right\rangle_{0}\right. \\
& -4\left\langle\left\langle E \gamma^{\alpha} \gamma^{\beta} \gamma^{\mu}\right\rangle_{0}\left\langle\left\langle\gamma_{\beta} \gamma_{\mu} E \gamma_{\alpha}\right\rangle\right\rangle_{0} .\right.
\end{aligned}
$$


Note that the first and the fourth terms on the right hand side are canceled with each other. Applying equation (15) to the second term, we have

$$
\left\langle\left\langle E \gamma^{\alpha} \gamma_{\alpha} \gamma^{\beta}\right\rangle\right\rangle_{0}\left\langle\left\langle\gamma_{\beta} E \gamma^{\mu} \gamma_{\mu}\right\rangle\right\rangle_{0}=\left\langle\left\langle\left\{\left(1-b_{1}\right) \Delta-G(\Delta)\right\} E \gamma^{\mu} \gamma_{\mu}\right\rangle\right\rangle_{0} .
$$

Using equation (15) again, we obtain

$$
\begin{aligned}
\left\langle\left\langle\Delta E \gamma^{\mu} \gamma_{\mu}\right\rangle_{0}\right. & =\left\langle\left\langle E \gamma^{\mu} \gamma_{\mu} \gamma^{\beta}\right\rangle\right\rangle_{0}\left\langle\left\langle\gamma_{\beta} \gamma^{\alpha} \gamma_{\alpha}\right\rangle_{0}\right. \\
& =\left\langle\left\langle\left\{\left(1-b_{1}\right) \Delta-G(\Delta)\right\} \gamma^{\alpha} \gamma_{\alpha}\right\rangle_{0} .\right.
\end{aligned}
$$

Moreover,

$$
\begin{aligned}
\left\langle\left\langle G(\Delta) \gamma^{\alpha} \gamma_{\alpha}\right\rangle_{0}\right. & =b_{\mu}\left\langle\left\langle\gamma^{\beta} \gamma_{\beta} \gamma^{\mu}\right\rangle\right\rangle_{0}\left\langle\left\langle\gamma_{\mu} \gamma^{\alpha} \gamma_{\alpha}\right\rangle_{0}\right. \\
& =\left\langle\left\langle\gamma^{\beta} \gamma_{\beta}\left\{\gamma^{\mu}-G\left(\gamma^{\mu}\right)\right\}\right\rangle\right\rangle_{0}\left\langle\left\langle\gamma_{\mu} \gamma^{\alpha} \gamma_{\alpha}\right\rangle_{0}\right. \\
& =\left\langle\left\langle\Delta \gamma^{\alpha} \gamma_{\alpha}\right\rangle_{0}-\left\langle\left\langle\gamma^{\beta} \gamma_{\beta} G(\Delta)\right\rangle\right\rangle_{0} .\right.
\end{aligned}
$$

Moving the second term on the right hand to the left hand, we obtain

$$
\left\langle\left\langle G(\Delta) \gamma^{\alpha} \gamma_{\alpha}\right\rangle_{0}=\frac{1}{2}\left\langle\left\langle\Delta \gamma^{\alpha} \gamma_{\alpha}\right\rangle_{0} .\right.\right.
$$

Hence, we have

$$
\left\langle\left\langle\Delta E \gamma^{\mu} \gamma_{\mu}\right\rangle_{0}=\left(\frac{1}{2}-b_{1}\right)\left\langle\left\langle\Delta \gamma^{\alpha} \gamma_{\alpha}\right\rangle_{0}\right.\right.
$$

By equation (19), we have

$$
\begin{aligned}
& \left\langle\left\langle E \gamma^{\alpha} \gamma_{\alpha} \gamma^{\beta}\right\rangle\right\rangle_{0}\left\langle\left\langle\gamma_{\beta} E \gamma^{\mu} \gamma_{\mu}\right\rangle_{0}\right. \\
= & \left(1-b_{1}\right)\left(\frac{1}{2}-b_{1}\right)\left\langle\left\langle\Delta \gamma^{\alpha} \gamma_{\alpha}\right\rangle_{0}-\left\langle\left\langle G(\Delta) E \gamma^{\mu} \gamma_{\mu}\right\rangle_{0} .\right.\right.
\end{aligned}
$$

To compute the last term on the right hand side of equation (18), we set

$$
f:=\left\langle\left\langle E \gamma^{\alpha} \gamma^{\beta} \gamma^{\mu}\right\rangle\right\rangle_{0}\left\langle\left\langle\gamma_{\beta} \gamma_{\mu} E \gamma_{\alpha}\right\rangle_{0}\right.
$$

Applying equation (7), we have

$$
\begin{aligned}
f & =\left\langle\left\langle\left\{G\left(\gamma^{\alpha}\right) \circ \gamma^{\mu}+\gamma^{\alpha} \circ G\left(\gamma^{\mu}\right)-G\left(\gamma^{\alpha} \circ \gamma^{\mu}\right)-b_{1} \gamma^{\alpha} \circ \gamma^{\mu}\right\} \gamma_{\mu} E \gamma_{\alpha}\right\rangle_{0}\right. \\
& =\left(2-b_{\alpha}-b_{\mu}-b_{1}\right)\left\langle\left\langle\left\{\gamma^{\alpha} \circ \gamma^{\mu}\right\} \gamma_{\mu} E \gamma_{\alpha}\right\rangle_{0}-\left\langle\left\langle G\left(\gamma^{\alpha} \circ \gamma^{\mu}\right) \gamma_{\mu} E \gamma_{\alpha}\right\rangle_{0}\right.\right. \\
& =\left(2-2 b_{\alpha}-b_{1}\right)\left\langle\left\langle\gamma^{\alpha} \gamma^{\mu} \gamma^{\beta}\right\rangle\right\rangle_{0}\left\langle\left\langle\gamma_{\beta} \gamma_{\mu} E \gamma_{\alpha}\right\rangle_{0}-\left\langle\left\langle\gamma^{\alpha} \gamma^{\mu} \gamma^{\beta}\right\rangle\right\rangle_{0}\left\langle\left\langle G\left(\gamma_{\beta}\right) \gamma_{\mu} E \gamma_{\alpha}\right\rangle_{0} .\right.\right.
\end{aligned}
$$

Switching $\alpha$ and $\beta$ in the last term, we have

$$
f=\left(2-b_{1}\right)\left\langle\left\langle E \gamma_{\alpha} \gamma_{\beta} \gamma^{\mu}\right\rangle\right\rangle_{0}\left\langle\left\langle\gamma_{\mu} \gamma^{\alpha} \gamma^{\beta}\right\rangle\right\rangle_{0}-3\left\langle\left\langle E G\left(\gamma_{\alpha}\right) \gamma_{\beta} \gamma^{\mu}\right\rangle_{0}\left\langle\left\langle\gamma_{\mu} \gamma^{\alpha} \gamma^{\beta}\right\rangle\right\rangle_{0} .\right.
$$


Applying equation (77) again, we have

$$
\begin{aligned}
& \left\langle\left\langle E \gamma_{\alpha} \gamma_{\beta} \gamma^{\mu}\right\rangle_{0}\left\langle\left\langle\gamma_{\mu} \gamma^{\alpha} \gamma^{\beta}\right\rangle\right\rangle_{0}\right. \\
= & \left\langle\left\langle\left\{G\left(\gamma_{\alpha}\right) \circ \gamma_{\beta}+\gamma_{\alpha} \circ G\left(\gamma_{\beta}\right)-G\left(\gamma_{\alpha} \circ \gamma_{\beta}\right)-b_{1} \gamma_{\alpha} \circ \gamma_{\beta}\right\} \gamma^{\alpha} \gamma^{\beta}\right\rangle\right\rangle_{0} .
\end{aligned}
$$

By the associativity of the quantum product and equation (13),

$$
\left\langle\left\langle\left\{G\left(\gamma_{\alpha}\right) \circ \gamma_{\beta}\right\} \gamma^{\alpha} \gamma^{\beta}\right\rangle\right\rangle_{0}=\left\langle\left\langle\left\{G\left(\gamma_{\alpha}\right) \circ \gamma^{\alpha}\right\} \gamma_{\beta} \gamma^{\beta}\right\rangle\right\rangle_{0}=\frac{1}{2}\left\langle\left\langle\Delta \gamma^{\alpha} \gamma_{\alpha}\right\rangle_{0}\right.
$$

and

$$
\begin{aligned}
\left\langle\left\langle G\left(\gamma_{\alpha} \circ \gamma_{\beta}\right) \gamma^{\alpha} \gamma^{\beta}\right\rangle\right\rangle_{0} & =\left\langle\left\langle\gamma_{\alpha} \gamma_{\beta} \gamma^{\mu}\right\rangle_{0}\left\langle\left\langle G\left(\gamma_{\mu}\right) \gamma^{\alpha} \gamma^{\beta}\right\rangle\right\rangle_{0}\right. \\
& =\left\langle\left\langle\gamma_{\alpha}\left\{G\left(\gamma_{\mu}\right) \circ \gamma^{\alpha}\right\} \gamma^{\mu}\right\rangle_{0}=\frac{1}{2}\left\langle\left\langle\Delta \gamma^{\alpha} \gamma_{\alpha}\right\rangle_{0}\right.\right.
\end{aligned}
$$

So we have

$$
\left\langle\left\langle E \gamma_{\alpha} \gamma_{\beta} \gamma^{\mu}\right\rangle_{0}\left\langle\left\langle\gamma_{\mu} \gamma^{\alpha} \gamma^{\beta}\right\rangle\right\rangle_{0}=\left(\frac{1}{2}-b_{1}\right)\left\langle\left\langle\Delta \gamma^{\alpha} \gamma_{\alpha}\right\rangle_{0}\right.\right.
$$

Moreover

$$
\begin{aligned}
& \left\langle\left\langle E G\left(\gamma_{\alpha}\right) \gamma_{\beta} \gamma^{\mu}\right\rangle_{0}\left\langle\left\langle\gamma_{\mu} \gamma^{\alpha} \gamma^{\beta}\right\rangle\right\rangle_{0}\right. \\
= & \left\langle\left\langle\left\{G\left(G\left(\gamma_{\alpha}\right)\right) \circ \gamma_{\beta}+G\left(\gamma_{\alpha}\right) \circ G\left(\gamma_{\beta}\right)-G\left(G\left(\gamma_{\alpha}\right) \circ \gamma_{\beta}\right)-b_{1} G\left(\gamma_{\alpha}\right) \circ \gamma_{\beta}\right\} \gamma^{\alpha} \gamma^{\beta}\right\rangle\right\rangle_{0} .
\end{aligned}
$$

Since

$$
\begin{aligned}
& \left\langle\left\langle\left\{G\left(\gamma_{\alpha}\right) \circ G\left(\gamma_{\beta}\right)\right\} \gamma^{\alpha} \gamma^{\beta}\right\rangle\right\rangle_{0} \\
= & \left\langle\left\langle\left\{G\left(\gamma_{\alpha}\right) \circ \gamma^{\alpha}\right\} G\left(\gamma_{\beta}\right) \gamma^{\beta}\right\rangle\right\rangle_{0}=\frac{1}{2}\left\langle\left\langle\left\{\gamma_{\alpha} \circ \gamma^{\alpha}\right\} G\left(\gamma_{\beta}\right) \gamma^{\beta}\right\rangle\right\rangle_{0} \\
= & \frac{1}{2}\left\langle\left\langle\gamma_{\alpha} \gamma^{\alpha}\left\{G\left(\gamma_{\beta}\right) \circ \gamma^{\beta}\right\}\right\rangle\right\rangle_{0}=\frac{1}{4}\left\langle\left\langle\Delta \gamma^{\alpha} \gamma_{\alpha}\right\rangle_{0}\right.
\end{aligned}
$$

and

$$
\begin{aligned}
& \left\langle\left\langle G\left(G\left(\gamma_{\alpha}\right) \circ \gamma_{\beta}\right) \gamma^{\alpha} \gamma^{\beta}\right\rangle\right\rangle_{0} \\
= & \left\langle\left\langle G\left(\gamma_{\alpha}\right) \gamma_{\beta} \gamma^{\mu}\right\rangle\right\rangle_{0}\left\langle\left\langle G\left(\gamma_{\mu}\right) \gamma^{\alpha} \gamma^{\beta}\right\rangle\right\rangle_{0}=\left\langle\left\langle\left\{G\left(\gamma_{\alpha}\right) \circ \gamma^{\mu}\right\} G\left(\gamma_{\mu}\right) \gamma^{\alpha}\right\rangle_{0}\right. \\
= & \left\langle\left\langle\left\{G\left(\gamma_{\alpha}\right) \circ \gamma^{\alpha}\right\} G\left(\gamma_{\mu}\right) \gamma^{\mu}\right\rangle_{0}=\frac{1}{2}\left\langle\left\langle\left\{\gamma_{\alpha} \circ \gamma^{\alpha}\right\} G\left(\gamma_{\mu}\right) \gamma^{\mu}\right\rangle_{0}\right.\right. \\
= & \frac{1}{2}\left\langle\left\langle\gamma_{\alpha} \gamma^{\alpha}\left\{G\left(\gamma_{\mu}\right) \circ \gamma^{\mu}\right\}\right\rangle_{0}=\frac{1}{4}\left\langle\left\langle\Delta \gamma^{\alpha} \gamma_{\alpha}\right\rangle_{0},\right.\right.
\end{aligned}
$$

together with equation (24), we have

$$
\left\langle\left\langle E G\left(\gamma_{\alpha}\right) \gamma_{\beta} \gamma^{\mu}\right\rangle_{0}\left\langle\left\langle\gamma_{\mu} \gamma^{\alpha} \gamma^{\beta}\right\rangle\right\rangle_{0}=\left(b_{\alpha}^{2}-\frac{1}{2} b_{1}\right)\left\langle\left\langle\Delta \gamma^{\alpha} \gamma_{\alpha}\right\rangle_{0} .\right.\right.
$$


Combining results of equations (23), (26) , and (29), we obtain that the last term on the right hand side of equation (18) is

$$
f=\left(-3 b_{\alpha}^{2}+b_{1}^{2}-b_{1}+1\right)\left\langle\left\langle\Delta \gamma^{\alpha} \gamma_{\alpha}\right\rangle_{0} .\right.
$$

Together with equation (22), we can simplify equation (18) as

$$
\begin{aligned}
G_{0}\left(E, E, \gamma^{\alpha}, \gamma_{\alpha}\right)= & \left\langle\left\langle E E \gamma^{\alpha} \gamma_{\alpha} \Delta\right\rangle_{0}-2\left\langle\left\langle G(\Delta) E \gamma^{\mu} \gamma_{\mu}\right\rangle\right\rangle_{0}\right. \\
& +\left(12 b_{\alpha}^{2}-2 b_{1}^{2}+b_{1}-3\right)\left\langle\left\langle\Delta \gamma^{\alpha} \gamma_{\alpha}\right\rangle_{0} .\right.
\end{aligned}
$$

On the other hand, by the definition of $\Phi$ in equation (16), we have

$$
\begin{aligned}
24 \Delta \Phi= & -\left\langle\left\langle\Delta E E \gamma_{\alpha} \gamma^{\alpha}\right\rangle_{0}-2\left\langle\left\langle\left\{\nabla_{\Delta} E\right\} E \gamma_{\alpha} \gamma^{\alpha}\right\rangle_{0}\right.\right. \\
& +12\left(b_{\alpha}\left(1-b_{\alpha}\right)-\frac{b_{1}+1}{6}\right)\left\langle\left\langle\Delta \gamma_{\alpha} \gamma^{\alpha}\right\rangle_{0} .\right.
\end{aligned}
$$

By equations (9) and (21), we have

$$
\begin{aligned}
\left\langle\left\langle\left\{\nabla_{\Delta} E\right\} E \gamma_{\alpha} \gamma^{\alpha}\right\rangle_{0}\right. & =\left\langle\left\langle\left\{-G(\Delta)+\left(b_{1}+1\right) \Delta\right\} E \gamma_{\alpha} \gamma^{\alpha}\right\rangle_{0}\right. \\
& =-\left\langle\left\langle G(\Delta) E \gamma_{\alpha} \gamma^{\alpha}\right\rangle_{0}+\left(b_{1}+1\right)\left(\frac{1}{2}-b_{1}\right)\left\langle\left\langle\Delta \gamma^{\alpha} \gamma_{\alpha}\right\rangle_{0} .\right.\right.
\end{aligned}
$$

Moreover,

$$
b_{\alpha}\left\langle\left\langle\Delta \gamma^{\alpha} \gamma_{\alpha}\right\rangle_{0}=\left\langle\left\langle\Delta \gamma^{\alpha} G\left(\gamma_{\alpha}\right)\right\rangle_{0}=\frac{1}{2}\left\langle\left\langle\Delta \gamma^{\alpha} \gamma_{\alpha}\right\rangle_{0}\right.\right.\right.
$$

So we have

$$
\begin{aligned}
24 \Delta \Phi= & -\left\langle\left\langle\Delta E E \gamma_{\alpha} \gamma^{\alpha}\right\rangle\right\rangle_{0}+2\left\langle\left\langle G(\Delta) E \gamma_{\alpha} \gamma^{\alpha}\right\rangle\right\rangle_{0} \\
& +\left(-12 b_{\alpha}^{2}+2 b_{1}^{2}-b_{1}+3\right)\left\langle\left\langle\Delta \gamma_{\alpha} \gamma^{\alpha}\right\rangle\right\rangle_{0} .
\end{aligned}
$$

Comparing with equation (31), we obtain

$$
G_{0}\left(E, E, \gamma^{\alpha}, \gamma_{\alpha}\right)=-24 \Delta \Phi
$$

The lemma is thus proved.

Proof of Theorem 1.1. Since $\Psi=\left\langle\left\langle E^{2}\right\rangle\right\rangle_{1}-\Phi$, this theorem follows from Lemmas [3.1, 3.2 and Equation (11).

\section{References}

[CX] Cox, D., and Katz, S., Mirror symmetry and algebraic geometry. Providence, R.I. AMS, 1999.

[DZ] Dubrovin, B., Zhang, Y., Bihamiltonian hierarchies in 2D topological field theory at oneloop approximation, Comm. Math. Phys. 198 (1998) 311 - 361.

[EHX] Eguchi, T., Hori, K., and Xiong, C., Quantum Cohomology and Virasoro Algebra, Phys. Lett. B402 (1997) 71-80. 
[Ge] Getzler, E., Intersection theory on $\bar{M}_{1,4}$ and elliptic Gromov-Witten Invariants, J. Amer. Math. Soc. 10 (1997) 973-998

$[\mathrm{K}]$ Kontsevich, M., Intersection theory on the moduli space of curves and the matrix airy function, Comm. Math. Phys., 147 (1992).

$[\mathrm{LiT}] \mathrm{Li}, \mathrm{J}$. and Tian, G., Virtual moduli cycles and Gromov-Witten invariants of general symplectic manifolds, Topics in symplectic 4-manifolds (Irvine, CA, 1996), 47-83.

[L1] Liu, X., Elliptic Gromov-Witten invariants and Virasoro conjecture. Comm. Math. Phys. 216 (2001), 705-728.

[L2] Liu, X., Genus-1 Virasoro conjecture on the small phase space, Symplectic Geometry and Mirror Symmetry, Preceedings of the 4th KIAS Annual International Conference, World Scientific, (2001), 265-279.

[LT] Liu, X., and Tian, G., Virasoro constraints for quantum cohomology. J. Diff. Geom. 50 (1998), $537-591$.

[RT] Ruan, Y. and Tian, G., Higher genus symplectic invariants and sigma models coupled with gravity, Invent. Math. 130 (1997), 455-516.

[W] Witten, E., Two dimensional gravity and intersection theory on Moduli space, Surveys in Diff. Geom., 1 (1991), 243-310.

\section{Xiaobo Liu}

Beijing International Center for Mathematical Research, Beijing University, Beijing, China.

\&

Department of Mathematics, University of Notre Dame,

Notre Dame, IN 46556, USA

Email: xliu3@nd.edu 